\title{
Produção sobre a assistência pré-natal nas dissertações e teses das ciências da saúde: estudo bibliométrico
}

\author{
Production on prenatal care in health science dissertations and theses: a bibliometric study
}

Producción sobre la atención prenatal en disertaciones y tesis de ciencias de la salud: estudio bibliométrico

\author{
Juliana de Castro Nunes Pereiral @; Nelson Miguel Galindo Neto" @ ; Guilherme Guarino de Moura Sáll! $\odot$;

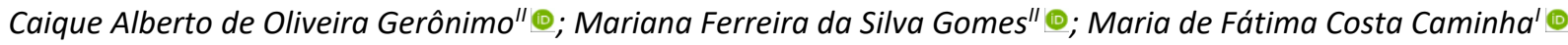 \\ IInstituto de Medicina Integral Professor Fernando Figueira, Recife, PE, Brasil; "Instituto Federal de Educação, Ciência e Tecnologia \\ de Pernambuco, Pesqueira, PE, Brasil; "'Instituto Federal de Educação, Ciência e Tecnologia de Pernambuco, Belo Jardim, PE, Brasil
}

\section{RESUMO}

Objetivo: descrever a produção científica brasileira das dissertações e teses das ciências da saúde acerca da assistência prénatal no Brasil. Método: estudo bibliométrico das produções disponibilizadas no Portal de Teses e Dissertações da Coordenação de Aperfeiçoamento de Pessoal de Nível Superior. Foram aplicadas as Leis de Lotka, Bradford e Zipf e estatística descritiva. Resultados: das 864 produções incluídas, 682 (78,9\%) eram dissertações, a maioria produzida na região Sudeste (44,6\%), por autores enfermeiros $(45,7 \%)$, orientadores médicos (39,9\%) e enfermeiros (32,5\%). Predominaram estudos descritivoexploratórios (75\%), com abordagem quantitativa (64\%), que investigaram a assistência/avaliação pré-natal (25\%), com as gestantes como população (66\%) e a Unidade Básica de Saúde como principal cenário (50,8\%). A palavra-chave mais frequente foi cuidado pré-natal. Conclusão: observou-se a predominância de estudos do tipo descritivo, quantitativos, oriundos das regiões Sudeste e Sul, nível de evidência 6.

Descritores: Bibliometria; Ciências da saúde; Gestantes; Cuidado Pré-Natal.

\begin{abstract}
Objective: to describe Brazilian scientific production in health science dissertations and theses on prenatal care in Brazil. Method: this bibliometric study examined the production available on the Theses and Dissertations Portal of Brazil's Coordination for the Improvement of Higher Education Personnel, applying Lotka, Bradford and Zipf Laws and descriptive statistics. Results: of the 864 productions included, 682 (78.9\%) were dissertations, most produced in the Southeast region $(44.6 \%)$, by nurse authors (45.7\%), medical and nursing advisors and nurses (39,9\% and $32.5 \%$, respectively). Descriptiveexploratory studies predominated (75\%), with quantitative approaches (64\%), investigating prenatal care/assessment (25\%), in populations of pregnant women (66\%) and with primary health care facilities as the main scenarios (50.8\%). The most frequent keyword was "prenatal care". Conclusion: the studies were predominantly descriptive, quantitative, from the Southeast and South regions and at evidence level 6.

Descriptors: Bibliometric; Health sciences; Pregnancy Women; Prenatal Care.

\section{RESUMEN}

Objetivo: describir la producción científica brasileña de disertaciones y tesis en ciencias de la salud sobre la atención prenatal en Brasil. Método: estudio bibliométrico de las producciones puestas a disposición en el Portal de Tesis y Disertaciones de la Coordinación para el Perfeccionamiento del Personal de Educación Superior. Se aplicaron las leyes Lotka, Bradford y Zipf y la estadística descriptiva. Resultados: de las 864 producciones incluidas, 682 (78,9\%) fueron disertaciones, la mayoría producidas en el Sureste (44,6\%), por enfermeras autoras (45,7\%), asesores médicos y de enfermaría (39,9\%, 32,5\% respectivamente). Predominaron los estudios descriptivos-exploratorios (75\%), con abordaje cuantitativo (64\%), que investigaron la atención / valoración prenatal (25\%), con gestantes como población (66\%) y la Unidad Básica de Salud como escenario principal (50,8\%). La palabra clave más frecuente fue atención prenatal. Conclusión: se observó el predomínio de estudios descriptivos, cuantitativos, provenientes de las regiones Sudeste y Sur, nivel de evidencia 6.

Descriptores: Bibliometría; Ciencias de la Salud; Mujeres embarazadas; Atención Prenatal.
\end{abstract}

\section{INTRODUÇÃO}

A assistência pré-natal trata-se do conjunto de medidas para assegurar o acompanhamento, desenvolvimento e observação dos aspectos da gestação. Para tanto, compreende a prevenção, identificação e correção de anormalidades maternas e/ou fetais, além de orientações sobre a gravidez, parto, puerpério e cuidados com recém-nascido ${ }^{1}$. A não realização ou realização inadequada dessa assistência é relacionada a maiores índices dos eventos potencialmente evitáveis de morbimortalidade materna e infantil².

As condutas adotadas no pré-natal devem ser baseadas em evidências científicas, que contribuam para a decisão dos profissionais de saúde. No Brasil, são necessários mais estudos que consolidem tais evidências ${ }^{3}$. Assim, o desenvolvimento de pesquisas direcionadas à assistência pré-natal, na área das Ciências da Saúde, é relevante, por possibilitar investigação referente aos aspectos inerentes à atuação multiprofissional. 
Dentre as fontes de produção científica, destacam-se os Programas de Pós-Graduação Stricto Sensu da área da saúde, que contribuem com a ciência, por meio da divulgação de relatórios de pesquisa na forma de dissertações e teses, que são requisitos para obtenção dos títulos de mestre e doutor ${ }^{4}$. Ao considerar que esses programas atuam como centros de construção de conhecimento, formação que estimula o pensamento crítico das questões inerentes à assistência à saúde, as dissertações e teses por eles produzidos refletem a visão, questionamentos, críticas e perspectivas da comunidade científica. Desse modo, torna-se relevante analisar os indicadores bibliométricos acerca da produção científica divulgada por meio de dissertações e teses da área da saúde, acerca da assistência pré-natal, no Brasil.

A partir dessa análise, será possível conhecer a evolução da produção ao longo dos anos, perceber as vertentes mais estudadas e conhecer caminhos metodológicos utilizados. Este estudo contribuirá para identificação de lacunas que poderão direcionar a realização de futuros estudos.

À vista disso, este estudo teve como objetivo descrever a produção científica brasileira das dissertações e teses das ciências da saúde acerca da assistência pré-natal no Brasil.

\section{REFERENCIAL TEÓRICO}

O Ministério da Saúde através do Programa de Humanização no Pré-natal e Nascimento preconiza, atividades que garantem o adequado acompanhamento pré-natal e assistência à gestante e a puérpera, sendo necessário a realização de exames laboratoriais, vacinação, atividades educativas e consultas pré-natal e puerpério ${ }^{5}$.

Contudo, apesar do aumento significativo do número de consultas pré-natal, persistem altos índices de mortalidade materna e neonatal por causas preveníveis, o que sugere falha deste serviço, apesar do reconhecimento prioritário dessas áreas já há algumas décadas ${ }^{6}$. Por conta da reconhecida vulnerabilidade no período gravídico, o pré-natal torna-se ferramenta essencial para mudar o perfil epidemiológico da saúde da mulher através da melhoria dessa assistência ${ }^{7}$.

Diante disso, o desenvolvimento de pesquisas na área do cuidado pré-natal surge como subsídio para incremento nas ações de saúde além de fornecer elementos de transformação na prática assistencial.

\section{MÉTODO}

Estudo bibliométrico, documental, com abordagem quantitativa. A bibliometria quantifica trabalhos científicos e mensura sua produção, além de permitir a recuperação da informação e acompanhamento do que é produzido em determinada área de estudo ${ }^{8,9}$.

A presente pesquisa foi realizada no período de março e abril de 2020, a partir de acesso online ao website do Portal de Teses e Dissertações da Coordenação de Aperfeiçoamento de Pessoal de Nível Superior (CAPES) para consulta às produções catalogadas no referido portal ${ }^{10}$. Não foi estabelecido limite temporal, entretanto, os textos completos encontravam-se disponíveis a partir de 2013. O material do estudo foi composto pelas dissertações e teses disponíveis no Portal da CAPES. Foram definidos os seguintes critérios de inclusão: ser dissertação ou tese da grande área de conhecimento Ciências da Saúde e abordar a assistência pré-natal. Os critérios de exclusão adotados foram não se encontrar disponível para acesso online e encontrar-se duplicado nas buscas.

Para busca dos estudos foi utilizado, individualmente, o descritor "Cuidado Pré-Natal", extraído a partir de consulta aos Descritores em Ciências da Saúde (DeCS). Além disso, utilizaram-se, em buscas distintas e separadamente, os descritores não controlados "Pré-Natal" e "Assistência Pré-natal”, extraídos a partir dos sinônimos do descritor controlado.

No levantamento bibliográfico, foram identificadas 2.144 produções científicas, destas, 199 foram encontradas a partir do descritor "Cuidado Pré-Natal", 260 do descritor "Assistência Pré-natal" e 1.685 do descritor "Pré-Natal”. Após leitura detalhada dos títulos e resumos, existiram 434 títulos duplicados, que foram contabilizados apenas uma vez e 846 foram excluídos por não abordarem o Pré-Natal. Assim, foram incluídas na amostra 864 produções científicas.

Destaca-se que a busca foi realizada por dois membros da equipe de pesquisa, de forma simultânea e independente. Os resultados obtidos foram comparados para identificação de contrassenso na identificação dos estudos, entre os pesquisadores.

Para registro dos dados de cada estudo selecionado, foi elaborado instrumento no software Microsoft Excel $2010^{\circ}$, que contemplou os indicadores bibliométricos: tipo de produção científica (tese ou dissertação); ano da produção; instituição e departamento dos quais a produção foi oriunda; Estado e região geográfica da instituição; categoria profissional do autor e do orientador; temática abordada; população e momento do ciclo reprodutivo estudados; local da coleta de dados; abordagem metodológica; tipo de estudo; nível de evidência e descritores/palavras-chave.

A definição dos níveis de evidência dos estudos ocorreu conforme classificação hierarquizada em sete níveis definidos: nível 1 - meta-análise ou revisões sistemáticas; nível 2 - ensaio clínico randomizado controlado; nível 3 - 
ensaio clínico sem randomização; nível 4 - estudos de coorte e de caso controle; nível 5 - revisões sistemáticas de estudos descritivos e qualitativos; nível 6 - estudos descritivos ou qualitativos; e nível 7 - opinião de especialistas ${ }^{11}$.

Os dados foram importados no software Statistical Package for the Social Sciences (SPSS) versão 21.0 e analisados por estatística descritiva (frequências absolutas e relativas).

Para análise, aplicou-se fundamentos de três leis da bibliometria ${ }^{12}$ : Os fundamentos da primeira lei, de Lotka, referente aos autores mais produtivos, foram contemplados ao considerar a quantidade de dissertações e teses orientadas por professor/orientador ${ }^{12,13}$. Os fundamentos da segunda lei, de Bradfort, referente ao veículo de divulgação da produção científica, foram contemplados mediante a mensuração, por instituições de ensino, dissertações e teses produzidas, o que possibilitou observar as instituições com maior e menor produção sobre o fenômeno estudado ${ }^{12}$. Os fundamentos da terceira lei, de Zipf, referente à contagem de frequência de palavras, foram contemplados a partir da mensuração dos descritores/palavras-chave das dissertações e teses ${ }^{12}$.

Por tratar-se de estudo com dados de acesso público, não houve necessidade da aprovação por Comitê de Ética em Pesquisa com Seres Humanos.

\section{RESULTADOS}

Das 864 produções científicas da amostra, 682 (78,9\%) eram dissertações e $182(21,1 \%)$ teses. Foram encontrados estudos disponíveis na íntegra de 2013 a 2019, sendo identificadas 91 (10,5\%) dissertações e 18 (2,1\%) teses em 2013, $93(10,8 \%)$ dissertações e 23 (2,6\%) teses em 2014, 102 (11,9\%) dissertações e 28 (3,2\%) teses em 2015, 108 (12,5\%) dissertações e 22 (2,5\%) teses em 2016, com pico de produção em 2017, onde foram identificadas 110 (12,7\%) dissertações e 33 (3,9\%) teses. No ano de 2018 foram registradas 101 (11,6\%) dissertações e 41 (4,8\%) teses e no ano seguinte 77 (8,9\%) dissertações e $17(2,0 \%)$ teses.

Observou-se que as dissertações e teses estavam vinculadas a 99 instituições, destas 37 (37,4\%) eram da região Sudeste, $25(25,2 \%)$ da Sul, $23(23,2 \%)$ da Nordeste, oito $(8,1 \%)$ da Centro-Oeste e seis $(6,1 \%)$ da Norte. Quanto ao número de estudos, por região geográfica, o Sudeste apresentou $385(44,6 \%)$ produções de dissertações e teses da área de Ciências da Saúde sobre pré-natal, com destaque para os Estados de São Paulo, com 207 (24,0\%) e Rio de Janeiro com 117 (13,5\%). A segunda região com maior quantidade de produções foi a Nordeste, com 232 (26,8\%), na qual os Estados que apresentaram maior produção foram Ceará, com 76 (8,8\%) e Pernambuco com 45 (5,2\%). Destaca-se ainda que, na região Nordeste, foram identificados os dois Estados com menor quantidade de produções: Alagoas com quatro $(0,5 \%)$ e Sergipe com três $(0,3 \%)$.

Na região Sul, o total de produções foi de 170 (19,6\%), com destaque para o Estado do Rio Grande do Sul, com $109(12,6 \%)$ e Paraná com 41 (4,7\%). No Centro-Oeste identificaram-se 53 (6,2\%) produções, com maior número nos Estado de Goiás, com 17 (2\%) e no Distrito Federal, com 16 (1,9\%). Na região Norte foram contabilizadas 24 (2,8\%) produções, com destaque para Amazonas, com nove (1\%) e Pará com oito $(0,9 \%)$.

Quanto a categoria administrativa das instituições, observou-se que 742 (85,9\%) eram públicas e 122 (14,1\%) privadas. A maioria, 576 (66,7\%), estava localizada na capital e 288 (33,3\%) no interior dos Estados. O detalhamento da produção de dissertações e teses, das instituições, por Estado e região, encontra-se na Tabela 1.

Foram identificadas 27 categorias profissionais relacionadas as autorias das dissertações e teses (Figura 1). Observouse que 395 (45,7\%) produções possuíram autores enfermeiros, 199 (23\%) médicos, 81 (9,4\%) nutricionistas, 51 (5,9\%) cirurgiões dentistas, 33 (3,8\%) fisioterapeutas, 28 (3,2\%) psicólogos, 23 (2,7\%) farmacêuticos e 13 (1,5\%) biomédico.

As dissertações e teses foram orientadas por pesquisadores de 24 categorias profissionais. Destas, destacaram-se 345 (39,9\%) orientadores médicos, 281 (32,5\%) enfermeiros, 61 (7,1\%) cirurgiões dentistas, 55 (6,4\%) nutricionistas, 33 (3,8\%) farmacêuticos, 20 (2,3\%) psicólogos e 13 (1,5\%) fisioterapeutas. As 56 (6,5\%) produções restantes foram orientadas por profissionais de 17 categorias distintas como, biólogos, profissionais de educação física, biomédicos, estatísticos e fonoaudiólogos.

Pelo critério de contagem direta, foram identificados 569 orientadores, responsáveis pelas 864 produções. Desses, $413(72,6 \%)$ orientaram apenas uma produção, cada; $84(14,7 \%)$ orientaram duas produções, 38 (6,6\%) orientaram três produções. Em contrapartida, apenas 34 (6,1\%) pesquisadores juntos, foram responsáveis por 169 orientações, que representou 19,6\% do total de orientações. Desses, 20 (3,5\%) orientaram quatro produções, 7 (1,2\%) cinco produções, $2(0,4 \%)$ seis produções, $2(0,4 \%)$ sete produções, $2(0,4 \%)$ oito produções e apenas $1(0,2 \%)$ orientou 12 produções.

Na grande área das Ciências da Saúde, o departamento de enfermagem apresentou maior número de produções sobre pré-natal, 202 (23,4\%); seguida por Saúde Coletiva, com 147 (17\%) produções; Ciências da Saúde, com 81 (9,4\%); Ciências Médicas/Medicina, com 46 (5,3\%); Saúde Pública, com 43 (5\%) e Nutrição, com 35 (4,1\%). As 310 (35,8\%) 
produções restantes foram oriundas de 37 departamentos distintos, das áreas da Saúde como tocoginecologia, saúde da mulher, criança e adolescente, saúde da família, saúde integral e odontologia.

TABELA 1: Distribuição da produção de dissertações e teses da área de Ciências da Saúde sobre pré-natal, segundo região e instituição, extraído do Portal de Teses e Dissertações CAPES no período de 2013-2019 ( $n=864)$. Brasil, 2020.

\begin{tabular}{|c|c|c|c|c|c|}
\hline \multirow[b]{2}{*}{ Região } & \multirow[b]{2}{*}{ Instituições com cinco ou mais produções } & \multirow{2}{*}{$\begin{array}{c}\text { Dissertação } \\
\mathbf{n}\end{array}$} & \multirow{2}{*}{$\begin{array}{c}\text { Tese } \\
\mathbf{n}\end{array}$} & \multicolumn{2}{|c|}{ Total } \\
\hline & & & & $\mathbf{n}$ & $\%$ \\
\hline \multirow[t]{3}{*}{ Norte } & Universidade Federal do Acre* & 6 & 0 & 6 & 0,7 \\
\hline & Universidade Federal do Pará* & 6 & 0 & 6 & 0,7 \\
\hline & Nota: 4 instituições com menos de 5 produções cada & 11 & 1 & 12 & 1,4 \\
\hline \multirow[t]{14}{*}{ Nordeste } & Universidade Federal do Maranhão* & 18 & 9 & 27 & 3,1 \\
\hline & Instituto de Medicina Integral Prof. Fernando Figueira** & 8 & 15 & 23 & 2,7 \\
\hline & Universidade Federal do Ceará* & 16 & 7 & 23 & 2,7 \\
\hline & Universidade de Fortaleza* & 21 & 0 & 21 & 2,4 \\
\hline & Universidade Estadual do Ceará* & 18 & 3 & 21 & 2,4 \\
\hline & Universidade Federal do Rio Grande do Norte* & 15 & 5 & 20 & 2,3 \\
\hline & Universidade Federal da Bahia* & 11 & 6 & 17 & 2,0 \\
\hline & Universidade Federal de Pernambuco* & 9 & 6 & 15 & 1,7 \\
\hline & Fundação Oswaldo Cruz (Fiocruz) ${ }^{*}$ & 11 & 0 & 11 & 1,3 \\
\hline & Universidade Estadual de Feira De Santana* & 10 & 0 & 10 & 1,2 \\
\hline & Universidade Federal do Piauí ${ }^{*}$ & 8 & 1 & 9 & 1,0 \\
\hline & Fiocruz (Centro de Pesquisa Aggeu Magalhães)* & 5 & 1 & 6 & 0,7 \\
\hline & Instituto de Ensino Superior do Piauí Ltda** & 5 & 0 & 5 & 0,6 \\
\hline & Nota: 4 instituições com menos de 5 produções cada & 20 & 4 & 24 & 2,7 \\
\hline \multirow[t]{5}{*}{ Centro-Oeste } & Universidade Federal de Goiás* & 14 & 3 & 17 & 2,0 \\
\hline & Universidade de Brasília* & 8 & 2 & 10 & 1,2 \\
\hline & Universidade Federal de Mato Grosso* & 9 & 1 & 10 & 1,2 \\
\hline & Universidade Federal de Mato Grosso do Sul* & 8 & 0 & 8 & 0,9 \\
\hline & Nota: 4 instituições com menos de 5 produções cada & 8 & 0 & 8 & 0,9 \\
\hline \multirow[t]{17}{*}{ Sudeste } & Universidade de São Paulo* & 29 & 17 & 46 & 5,3 \\
\hline & Universidade de São Paulo (Ribeirão Preto)* & 26 & 13 & 39 & 4,5 \\
\hline & Fundação Oswaldo Cruz (Fiocruz)* & 29 & 9 & 38 & 4,4 \\
\hline & Universidade Estadual de Campinas* & 26 & 10 & 36 & 4,2 \\
\hline & Universidade Federal do Rio de Janeiro* & 25 & 7 & 32 & 3,7 \\
\hline & Universidade Federal de Minas Gerais* & 22 & 4 & 26 & 3,0 \\
\hline & Universidade Federal Fluminense* & 26 & 0 & 26 & 3,0 \\
\hline & Universidade Estadual Paulista Júlio de Mesquita Filho (Botucatu)* & 14 & 7 & 21 & 2,4 \\
\hline & Universidade Federal de São Paulo* & 10 & 8 & 18 & 2,1 \\
\hline & Universidade Federal do Espírito Santo* & 15 & 1 & 16 & 1,9 \\
\hline & Universidade do Estado do Rio De Janeiro* & 8 & 2 & 10 & 1,2 \\
\hline & Universidade Estácio de Sá** & 7 & 0 & 7 & 0,8 \\
\hline & Centro Universitário Saúde $A b c^{* *}$ & 3 & 3 & 6 & 0,7 \\
\hline & Universidade Federal de Juiz de Fora* & 6 & 0 & 6 & 0,7 \\
\hline & Universidade Federal de São Carlos* & 6 & 0 & 6 & 0,7 \\
\hline & Universidade Estadual Paulista Júlio de Mesquita Filho (Araçatuba) * & 5 & 0 & 5 & 0,6 \\
\hline & Nota: 21 instituições com menos de 5 produções cada & 44 & 3 & 47 & 5,4 \\
\hline \multirow[t]{11}{*}{ Sul } & Universidade Federal do Rio Grande do Sul* & 24 & 4 & 28 & 3,2 \\
\hline & Universidade Federal do Rio Grande* & 14 & 4 & 18 & 2,1 \\
\hline & Universidade Estadual de Maringá* & 12 & 4 & 16 & 1,9 \\
\hline & Universidade Federal de Pelotas* & 6 & 9 & 15 & 1,7 \\
\hline & Universidade Federal de Santa Catarina* & 9 & 6 & 15 & 1,7 \\
\hline & Universidade Franciscana** & 15 & 0 & 15 & 1,7 \\
\hline & Universidade Federal do Paraná* & 13 & 0 & 13 & 1,5 \\
\hline & Universidade Federal de Santa Maria* & 10 & 1 & 11 & 1,3 \\
\hline & Universidade Católica de Pelotas ${ }^{* *}$ & 7 & 2 & 9 & 1,0 \\
\hline & Universidade Estadual de Londrina* & 4 & 1 & 5 & 0,6 \\
\hline & Nota: 15 instituições com menos de 5 produções cada & 22 & 3 & 25 & 2,9 \\
\hline
\end{tabular}

*Instituição Pública; ${ }^{* *}$ Instituição Privada 


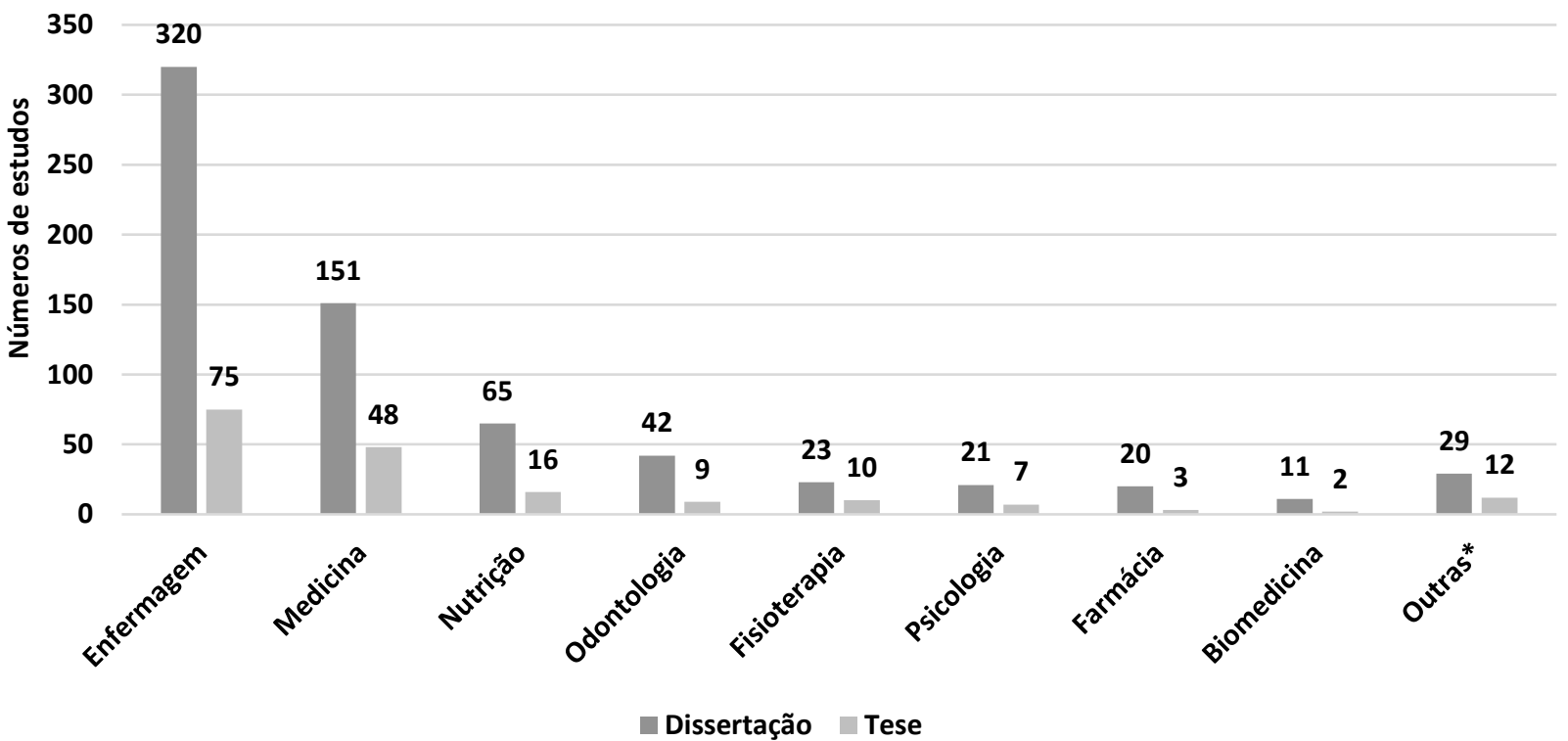

FIGURA 1: Distribuição da produção de dissertações e teses da área de Ciências da Saúde sobre pré-natal, por categoria profissional do autor. Recife, Pernambuco, Brasil, 2020.

*Outras - Ciências Biológicas; Educação Física; Serviço Social; Terapia Ocupacional; Pedagogia; Saúde Pública; Fonoaudiologia; Ciências Sociais Química; Estatística; Física; Engenharia Elétrica; Informática Biomédica; Ciências da Computação; Matemática; Medicina Veterinária; Análise e Desenvolvimento de Sistemas; Jornalismo; Biotecnologia

Ao verificar o delineamento metodológico, identificou-se que 553 (64\%) tiveram abordagem quantitativa, 218 $(25,2 \%)$ qualitativa e as pesquisas com abordagem mista (quanti-qualitativas) corresponderam a 93 (10,8\%). A maioria dos estudos, 629 (72,8\%), foi transversal, seguido por 106 (12,3\%) coorte, 49 (5,7\%) caso-controle, 28 (3,2\%) metodológico, 21 (2,4\%) experimental, $16(1,9 \%)$ revisão de literatura e $15(1,7 \%)$ quase-experimental. No tocante ao nível de evidência, identificou-se que 648 (75\%) dos estudos possuíam evidência nível 6, 156 (18,1\%) nível 4, 25 (2,8\%) nível 2, $18(2,1 \%)$ nível 3, dez $(1,2 \%)$ nível 1 e sete $(0,8)$ apresentaram nível de evidência 5 .

Foi possível observar que as dissertações e teses contemplaram 26 subtemas, acerca da assistência pré-natal, que variaram desde os distintos momentos do ciclo gravídico-puerperal, até agravos à saúde materna e fetal. Dentre as populações estudadas nas dissertações e teses sobre pré-natal, observou-se 20 arranjos de tipos de públicos que foram alvo das pesquisas, que contemplaram o binômio mãe-filho, acompanhantes/parceiros, estudantes e profissionais de saúde, conforme observado na Tabela 2.

Dos 864 estudos, observou-se que a Unidade Básica de Saúde (UBS) foi cenário de 437 (50,8\%), os serviços hospitalares de 367 (42,5\%), os bancos de informação de 40 (4,6\%), universidades de cinco (0,5\%), Centros de Atenção Psicossocial (CAPS) de três $(0,3 \%)$, penitenciárias de três $(0,3 \%)$ e em nove $(1,0 \%)$ não foi especificado o setor onde o estudo foi conduzido.

A partir da aplicação da Lei de Zipf, foi possível construir lista das palavras-chaves cadastradas nas dissertações e teses. Foram encontradas 3745 formas (palavras-chave), com 1329 ocorrências. As palavras-chave com maior frequência foram "Cuidado Pré-natal” com 197 (14,9\%) ocorrências, seguida por "Gestante" com 134 (10\%), "Gravidez" com 125 (9,4\%), "Gestação" com 96 (7,2\%), "Pré-natal" com 93 (7\%) e "Enfermagem" com 76 (5,7\%). As demais, 608 $(45,8 \%)$ palavras contemplaram agravos à saúde, como sífilis, diabetes mellitus gestacional, pré-eclâmpsia, obesidade, depressão, ansiedade, anemia, zika vírus e toxoplasmose. 
TABELA 2: Subtemas e população estudada das dissertações e teses da área de Ciências da Saúde sobre pré-natal extraído do Portal de Teses e Dissertações CAPES no período de 2013-2019. Brasil, 2020. (n=864)

\begin{tabular}{|c|c|c|c|c|}
\hline \multirow{2}{*}{ Variável } & \multirow{2}{*}{$\begin{array}{c}\text { Dissertações } \\
\mathbf{n}\end{array}$} & \multirow{2}{*}{$\begin{array}{c}\text { Teses } \\
\mathbf{n}\end{array}$} & \multicolumn{2}{|c|}{ Total } \\
\hline & & & $\mathbf{n}$ & $\%$ \\
\hline \multicolumn{5}{|l|}{ Subtemas } \\
\hline Assistência (qualidade/adequação/avaliação) & 175 & 41 & 216 & 25,0 \\
\hline Infecções sexualmente transmissíveis & 98 & 14 & 112 & 13,0 \\
\hline Nutrição e alimentação (sobrepeso e obesidade, vitamina, IMC*) & 36 & 20 & 56 & 6,5 \\
\hline Doença crônica (DM**/HAS***/Hipotireoidismo/Anemia/Hepatite) & 44 & 12 & 56 & 6,5 \\
\hline Gravidez na adolescência & 46 & 9 & 55 & 6,4 \\
\hline Infecção/doença infecciosa & 35 & 9 & 44 & 5,1 \\
\hline Parto & 31 & 8 & 39 & 4,5 \\
\hline Saúde bucal e digestiva & 33 & 4 & 37 & 4,3 \\
\hline Risco a saúde materna & 28 & 5 & 33 & 3,8 \\
\hline Álcool e outras drogas & 19 & 8 & 27 & 3,1 \\
\hline Filho: feto/neonato/recém-nascido & 21 & 6 & 27 & 3,1 \\
\hline Saúde mental da gestante & 16 & 8 & 24 & 2,8 \\
\hline Doenças hipertensivas: Eclâmpsia/Heelp & 12 & 5 & 17 & 2,0 \\
\hline Violência contra gestante/mãe & 12 & 5 & 17 & 2,0 \\
\hline Amamentação (aleitamento/mama) & 12 & 2 & 14 & 1,6 \\
\hline Arbovirose (zika, dengue, chykungunya) & 10 & 3 & 13 & 1,5 \\
\hline Tratamento farmacológico & 10 & 3 & 13 & 1,5 \\
\hline Doença congênita & 10 & 1 & 11 & 1,3 \\
\hline Pai, companheiro e família & 6 & 5 & 11 & 1,3 \\
\hline Exames laboratoriais e de imagem & 7 & 4 & 11 & 1,3 \\
\hline Exercício físico (estilo de vida) & 5 & 4 & 9 & 1,0 \\
\hline Sexualidade na gravidez/pós-parto & 6 & 1 & 7 & 0,8 \\
\hline Câncer & 3 & 2 & 5 & 0,6 \\
\hline Exame ginecológico & 2 & 2 & 4 & 0,4 \\
\hline Lúpus eritematoso sistêmico & 3 & 1 & 4 & 0,4 \\
\hline Distúrbio de sono na gestação & 2 & 0 & 2 & 0,2 \\
\hline \multicolumn{5}{|l|}{ População estudada } \\
\hline Gestantes & 407 & 103 & 510 & 59,0 \\
\hline Mães: puérperas/ após puerpério & 85 & 22 & 107 & 12,4 \\
\hline Equipe multiprofissional ${ }^{* * * *}$ & 73 & 5 & 78 & 9,1 \\
\hline Mãe e filho & 45 & 14 & 59 & 6,8 \\
\hline Gestantes e profissionais & 20 & 13 & 33 & 3,8 \\
\hline Gestantes e puérperas & 13 & 7 & 20 & 2,3 \\
\hline Filho: recém-nascido/criança & 16 & 4 & 20 & 2,3 \\
\hline Acompanhante/Parceiro/companheiro & 8 & 3 & 11 & 1,4 \\
\hline Gestores e profissionais & 3 & 2 & 5 & 0,6 \\
\hline Nascidos mortos & 2 & 2 & 4 & 0,5 \\
\hline Gestantes e mulheres não gestantes & 0 & 4 & 4 & 0,5 \\
\hline Parteiras & 3 & 0 & 3 & 0,3 \\
\hline Gestantes e Gestores & 0 & 2 & 2 & 0,2 \\
\hline Estudantes de saúde & 2 & 0 & 2 & 0,2 \\
\hline Mãe, filho e enfermeiro & 1 & 0 & 1 & 0,1 \\
\hline Parceiros e profissionais & 0 & 1 & 1 & 0,1 \\
\hline Gestante e Profissional Educ. Física & 1 & 0 & 1 & 0,1 \\
\hline Gestantes e parceiros & 1 & 0 & 1 & 0,1 \\
\hline Puérperas e profissionais & 1 & 0 & 1 & 0,1 \\
\hline Puérpera e acompanhante & 1 & 0 & 1 & 0,1 \\
\hline
\end{tabular}

*IMC - Índice de Massa Corporal; ${ }^{* *} \mathrm{DM}$ - Diabetes Mellitus; ${ }^{* * *} \mathrm{HAS}$ - Hipertensão Arterial Sistêmica. ${ }^{* * * *}$ médico, enfermeiro, cirurgião dentista, agente comunitário de saúde, nutricionista, profissional de educação física.

\section{DISCUSSÃo}

Ao descrever a produção científica brasileira das dissertações e teses das Ciências das Saúde acerca da assistência pré-natal, o estudo atual encontrou que as instituições que apresentaram maior quantitativo de produção acadêmica pertenciam às regiões Sudeste e Sul. Resultado semelhante foi encontrado em análise bibliométrica que identificou que 
essas regiões produziram maior número de estudos sobre Unidade de Terapia Intensiva ${ }^{4}$. Esse resultado pode estar associado ao fato dessas regiões serem pioneiras na implementação de cursos stricto sensu na área de saúde. Em contrapartida, a maior parte dos trabalhos foram produzidos em Programas de Pós-Graduação da região Sudeste e Nordeste. Esse achado corrobora resultados de estudo bibliométrico sobre enfermagem em cuidado paliativo, em que essas regiões apresentaram maior produção acadêmica ${ }^{14}$. Isto se deve, possivelmente, a presença de instituições e grupos de pesquisa de referência nas investigações sobre a temática analisada. Ao considerar que o Brasil possui dimensões continentais, com especificidades em cada região que demandam pesquisas, sugere-se, o incremento de investimentos para estímulo de produções nas demais regiões.

Quanto a categoria administrativa, a maioria eram universidades públicas. Essa informação é corroborada por levantamento feito na Web of Science Group, em que 15 universidades públicas foram responsáveis por mais de 60\% do conhecimento científico produzido no País ${ }^{15}$. Este dado pode ser justificado diante da expressiva atuação das instituições públicas, alicerçada na tríade ensino, pesquisa e extensão e elevada qualidade dos pesquisadores que atuam em tais instituições. Além disso, esses centros são o berço da formação Stricto Sensu, de forma que são responsáveis por elevada quantidade das produções científicas.

A maioria das dissertações e teses foi oriunda dos departamentos de enfermagem e os enfermeiros correspondiam à maioria dos autores das produções. Resultado semelhante foi observado em estudo sobre segurança do paciente em Unidade de Terapia Intensiva, em que essa categoria profissional representou $85 \%$ dos autores ${ }^{16}$. Esses achados são fruto da expansão da atuação da enfermagem que, além de exercer atividade na assistência à saúde, tem contribuído, de forma crescente, mediante vinculação a grupos de pesquisa e engajamento em Programas de PósGraduação, com o desenvolvimento de pesquisas para avanço científico que direcione sua atuação profissional ${ }^{17}$. Tal fato possui relevância social, uma vez que a pesquisa e investigação em enfermagem são consideradas essenciais para assistência qualificada, pois a geração de estudos e a integração de práticas baseada em evidências influenciam e melhoram a atuação da categoria que possui maior quantitativo de profissionais na saúde, a enfermagem ${ }^{18}$.

Os indicadores bibliométricos apontaram maior número de dissertações. Resultados semelhantes foram encontrados em outro estudo, que analisou a produção dos Programas de Pós-Graduação em Enfermagem acerca do serviço de atendimento móvel de urgência identificou que as dissertações de mestrado corresponderam ao maior número de estudos ${ }^{9}$. Estes dados justificam-se, pois, os cursos de mestrado são vistos predominantemente como primeiro degrau para qualificação acadêmico-científica, ademais, são mais numerosos e foram os primeiros a serem implantados. No entanto, com o progresso na atividade científica e tecnológica no Brasil, nos últimos anos, observa-se também número crescente de cursos de doutorado. Assim, espera-se que as teses deles oriundas representem maior quantitativo nos indicadores bibliométricos de futuros estudos ${ }^{19}$.

Acerca dos orientadores, destacaram-se os profissionais médicos, seguido por enfermeiros. O elevado quantitativo de orientadores médicos pode ter relação com o fato dos primeiros grupos de pesquisa criados no Brasil, terem surgido nos departamentos de Medicina Preventiva e Social ${ }^{20}$. Além disso, a predominância de orientadores médicos e enfermeiros pode justificar-se uma vez que estes pesquisadores também se encontram inseridos em Programas de Pós-Graduação de diversas modalidades das ciências da saúde. No entanto, cabe destacar a importância de outros profissionais da saúde participarem de investigações sobre o pré-natal, com vista a contemplar as perspectivas dos demais integrantes da equipe multiprofissional, sobre a temática.

No tocante a produtividade dos orientadores, poucos pesquisadores foram responsáveis pela maior parte das orientações, ao passo que a maioria orientou pequeno número de estudos. Isto vai de encontro à Lei de Lotka, que afirma que poucos autores são responsáveis por aproximadamente $60 \%$ da produção científica, enquanto o maior número de autores contribui com poucas produções em um determinado campo científico ${ }^{13}$. Destaca-se que a produtividade dos professores-orientadores, é influenciada pelo desenvolvimento de estudos do seu grupo de pesquisa, perfil dos seus orientandos, disputa e contemplação em editais de financiamento e transversalidade de tema sobre o qual pesquise, com o pré-natal.

No que concerne à abordagem metodológica, observou-se tendência para realização de pesquisas quantitativas, que também foi encontrada em revisão sobre assistência pré-natal ${ }^{21}$. Tal achado é relevante pois os estudos quantitativos permitem que, em pesquisas sobre o pré-natal, aspectos importantes sejam investigados, diante da possibilidade de mensurar variáveis numéricas, estabelecer análise de associações/correlações estatísticas, que podem explicar fenômenos na saúde. Apesar disto, torna-se urgente investigações com abordagem qualitativa, para revelar aspectos subjetivos, que não são verificados por meio de estudo quantitativo e que são relevantes para tomada de decisão na assistência em saúde. 
O tipo de estudo que prevaleceu foi o descritivo (nível de evidência 6). Embora relevante, esse tipo de estudo se restringe a descrever a ocorrência de eventos. Com isto, observa-se a necessidade de outras pesquisas com nível de evidência superior, que forneçam dados sobre causa-efeito e analisem intervenções na área de pré-natal.

Mesmo com a diversidade de temas investigados nas dissertações e teses, houve maior ênfase em pesquisas sobre "qualidade, adequação e avaliação pré-natal”, seguida por "infecções sexualmente transmissíveis (IST)". Investigar a qualidade é relevante, pois, impacta diretamente nas taxas de prematuridade e morbimortalidade materna-infantil. Em estudo realizado na Etiópia, sobre qualidade pré-natal, foi possível observar como a baixa assistência influencia na saúde da gestante e que medidas como aumento da infraestrutura, consulta pré-natal direcionada e incentivo à educação materna, implicam em diminuição dos gastos de saúde e melhoram a qualidade da assistência ${ }^{22}$. No que tange às IST, estas evidenciam-se por serem grave problema para a saúde pública, que representa a segunda causa na procura por atendimento nos serviços de saúde, com alta prevalência entre as gestantes ${ }^{23}$, de forma que são alvos relevantes de investigação científica.

No tocante à população estudada, destacaram-se as gestantes, o que é esperado diante do objetivo envolver prénatal. Entretanto, cabe apontar que o ciclo gravídico-puerperal envolve dois sujeitos, a gestante e o feto. Logo, é pertinente que mais estudos abordem os aspectos relacionados à saúde fetal, durante o pré-natal. Em revisões sistemáticas que abordaram mãe-filho no ciclo gravídico-puerperal, com busca mundial, não foram observados estudos brasileiros ${ }^{24,25}$. Além do filho, é importante que sejam realizados mais estudos com os outros atores envolvidos na rede de apoio à gestante, como parceiro, familiares e profissionais. Pesquisas nacionais demonstraram que a presença do acompanhante vem aumentando na assistência à gestante e ao parto, entretanto, observou-se ainda baixo acesso à informação o que limita essas boas práticas assistenciais ${ }^{26,27}$. Ressalta-se com isto, a necessidade da produção de dissertações e teses que envolvam esses atores.

O principal cenário de estudo nas produções científicas foi a Unidade Básica de Saúde (UBS), considerada porta de entrada das gestantes no Sistema Único de Saúde (SUS), além de ser espaço voltado para prevenção, que contempla em suas atividades preconizadas o estabelecimento da consulta pré-natal. Assim, é pertinente o interesse dos pesquisadores em desenvolver estudos nesse ambiente da atenção primária. Observa-se, no entanto, a necessidade de estudos que realizem investigações em outros cenários, como intercorrências gestacionais atendidas por unidades hospitalares e instituições de referência para pré-natal de alto risco.

Nas produções analisadas, as palavras-chave remeteram, em sua maioria, ao cuidado pré-natal, além daquelas relacionadas à gestação, enfermagem e agravos à saúde gestacional. Observa-se, portanto, que as palavras traduziram, sobretudo, o momento do ciclo-gravídico investigado, a categoria profissional e os agravos investigados. Desse modo, aponta-se a importância da decisão criteriosa das palavras-chave de um trabalho acadêmico, visto que elas devem traduzir, com clareza, a essência do estudo e, assim, facilitar a localização de conteúdos específicos durante buscas na literatura.

O estudo apontou a caracterização de dissertações e teses e, com isso, revelou lacunas de perfil de produção, de população estudada e de tipos de estudos. Esses resultados podem direcionar pesquisadores da área de pré-natal, para contemplar as lacunas identificadas em futuros estudos. Além disso, o apontamento das instituições com maior produção pode viabilizar parcerias interinstitucionais, bem como a realização de pesquisas multicêntricas, acerca da temática.

\section{Limitações do estudo}

Aponta-se como limitação do estudo atual, a possibilidade da perda de produções elegíveis no Portal de Teses e Dissertações da CAPES, uma vez que o tempo entre a defesa e o registro da produção dependem de trâmites burocráticos nas instituições de ensino para disponibilização online no portal.

Destaca-se ainda, que esta pesquisa verificou apenas dissertações e teses das Ciências da Saúde, de forma que os achados encontrados podem não corresponder à realidade de produções que envolvam pré-natal em outras áreas de investigação.

\section{CONCLUSÃO}

Os indicadores bibliométricos das dissertações e teses da área das Ciências da Saúde sobre pré-natal permitiram constatar a predominância de estudos do tipo descritivo, com nível de evidência 6, quantitativos, oriundos das regiões Sudeste e Sul, que tratavam sobre assistência pré-natal e apresentavam como população investigada a gestante.

Atestou-se predominância de estudos oriundos de departamentos de enfermagem, com enfermeiros e médicos como os autores e orientadores da maior parte das produções. Diante do exposto, recomenda-se pesquisas nas diversas regiões do país e que contemplem outras abordagens metodológicas. 


\section{REFERÊNCIAS}

1. Lima KMSG, Santos HJ, Pereira J, Barbosa LP, Cabral MCAM, Silva P, et al. Nursing Care at High Risk Prenatal Care. Brazilian Journal of health Review. [Internet]. 2019 [cited 2020 May 02]; 2(4):3183-97. Available from: http://www.brazilianjournals.com/index.php/BJHR/article/view/2173

2. Santos MAM, Miranda MIF, Nunes DD. Perfil epidemiológico da mortalidade neonatal precoce, no período de 2008 a 2015 , em Porto Velho, Rondônia, Brasil. Sodebrás [Internet]. 2018 [cited 2020 May 02]; 13(151):83-7. Available from: http://www.sodebras.com.br/edicoes/N151.pdf

3. Nascimento JS, Silva MR, Oliveira ECT, Monte GCSB. Assistance to Women in Prenatal, Childbirth and Birth: Contributions from the Stork Network. Revist. Port.: Saúde e Sociedade. [Internet]. 2018 [cited 2020 May 05]; 3(1):694-709. Available from: https://www.seer.ufal.br/index.php/nuspfamed/article/download/4241/4532

4. Santos MAB, Sá GGM, Caetano JÁ, Costa AB, Muniz MLC, Galindo Neto NM. Brazilian nursing dissertations and theses conducted in intensive care units. Rev Rene. [Internet]. 2017 [cited 2020 May 05];18(4):521-7. DOI: https://doi.org/10.15253/2175-6783.2017000400014

5. Brasil. Brasil. Ministério da Saúde. Secretaria de Políticas de Saúde. Área Técnica da Saúde da Mulher. Programa de Humanização no Pré-natal e Nascimento. [Internet] 2002 [cited 2020 Nov 04]. Available from: https://bvsms.saude.gov.br/bvs/publicacoes/parto.pdf

6. Ruas CAM, Quadros JFC, Rocha JFD, Rocha FC, Andrade Neto GR, Piris AP, et al. Perfil e distribuição espacial da mortalidade materna. Rev. Bras. Saude Mater. Infant. [Internet]. 2020 [cited 2020 Nov 04]; 20(2):1-13. Available from: https://doi.org/10.1590/1806-93042020000200004

7. Martins ACS, Silva LS. Epidemiological profile of maternal mortality. Rev. Bras. Enferm. [Internet]. 2018 [cited 2020 Nov 04]; 71(Suppl 1):677-83. DOI: http://dx.doi.org/10.1590/0034-7167-2017-0624

8. Garcia LAA, Camargo FC, Gomes THM, Rezende MP, Pereira GA, Iwamoto HH, et al. Production of knowledge of Nursing about potential life years lost: bibliometric study. REFACS (online). [Internet]. 2017 [cited 2020 May 08];5(1):34-46. DOI: http://dx.doi.org/10.18554/refacs.v5i1.1911

9. Agra MAC, Freitas TCS, Caetano JA, Alexandre ACS, Sá GGM, Galindo Neto NM. Nursing dissertations and theses on the mobile emergency care services: a bibliometric study. Texto Contexto Enferm. [Internet]. 2018 [cited 2020 May 08]; 27(1):1-10. DOI: https://doi.org/10.1590/0104-07072018003500016

10. Brasil, Coordenação de Aperfeiçoamento de Pessoal de Nível Superior-Capes. Banco de Teses e Dissertações [Internet]. 2016 [cited 2020 May 08]. Available from: http://bancodeteses.capes.gov.br/

11. Melnyk BM, Fineout-Overholt E. Making the case for evidence-based practice. In: Melnyk BM, FineoutOverholt E. Evidencebased practice in nursing \& healthcare: a guide to best practice. Philadelphia (US): Lippincott Williams \& Wilkins; 2005. p.3-24.

12. Machado Junior C, Souza MTS, Parisotto IRS, Palmisano A. The Laws of Bibliometrics in Different Scientific Databases. Revista de Ciências da Administração. [Internet]. 2016 [cited 2020 May 09];18(44):111-23. DOI: http://dx.doi.org/10.5007/21758077.2016v18n44p111

13. Cândido RB, Garcia FG, Campos ALS, Tambosi Filho E. Lotka's Law: a look at the productivity of the authors in the brazilian literature of finance. Revista eletrônica de biblioteconomia e ciência da informação. [Internet]. 2018 [cited 2020 May 09]; 23(53):1- 15. DOI: http://dx.doi.org/10.5007/1518-2924.2018v23n53p1

14. Almeida ARLP, Oliveira FA, Marinho CLA, Leite AMC, Silva RS. Nursing in palliative care in dissertations and theses in brazil: a bibliometric study. Rev Min Enferm. [Internet]. 2019 [cited 2020 May 19]; 23:1-8. DOI: https://doi.org/10.5935/14152762.20190036

15. Web of Science Group. Research in Brazil: Funding excellence. Clarivate Analytics company. [Internet]. 2019 [cited 2020 May 19]; 1-42. Available from: https://jornal.usp.br/wp-content/uploads/2019/09/ClarivateReport_2013-2018.pdf

16. Oliveira BKF, Silva MA, Carvalho AS, Santos FF. Segurança do paciente em unidade de terapia intensiva: estudo bibliométrico. Revista Eletrônica Acervo Enfermagem. [Internet]. 2020 [cited 2020 May 12]; 3:1-8. DOI: https://doi.org/10.25248/reaenf.e2724.2020

17. Ribeiro RP, Marziale MHP. Editorial characteristics and quality of the articles published by Brazilian Nursing journals. Rev Esc Enferm USP. [Internet]. 2018 [cited 2020 May 21]; 52:1-7. DOI: http://dx.doi.org/10.1590/S1980-220X2017030003367

18. Ives E, Jeanette DNP, Pappas, Sharon. The Value of Nursing Research, JONA: The Journal of Nursing Administration. [Internet]. 2020 [cited 2020 May 19]; 50(5):243-4. DOI: https://doi.org/10.1097/NNA.0000000000000876

19. Soares PC. Contradições na pesquisa e pós-graduação no Brasil. Estud. Av. [Internet]. 2018 [cited 2020 May 19]; 32(92):289313. DOI: https://doi.org/10.5935/0103-4014.20180020

20. Cruz MM, Oliveira SRA, Campos RO. Research groups of health evaluation in Brazil: an overview of collaborative networks. SAÚDE Debate. [Internet]. 2019 [cited 2020 May 19]; 43(122):657-67. Available from: https://www.scielo.br/pdf/sdeb/v43n122/0103-1104-sdeb-43-122-0657.pdf

21. Cruz GC, Ruiz PC, Ribeiro Junior OC, Sousa AD, Pereira RMO, Barroso CO, et al. Métodos de avaliação da qualidade de assistência ao pré-natal no Brasil: revisão integrativa da literatura [Internet]. 2019 [cited 2020 May 14]; 27:1-8. DOI: https://doi.org/10.25248/reas.e521.2019

22. Kassaw A, Debie A, Geberu DM. Quality of Prenatal Care and Associated Factors among Pregnant Women at Public Health Facilities of Wogera District, Northwest Ethiopia. Journal of Pregnancy. [Internet]. 2020 [cited 2020 May 20]; 2020:1-8. DOI: https://doi.org/10.1155/2020/9592124 
23. Ricci AP, Sene AG, Souza BLB, Aguiar KM, Figueiredo LR, Gerk MA. Sexually transmitted infections during pregnancy: health education as a prevention strategy in primary care. Braz. J. Hea. Rev. [Internet]. 2019 [cited 2020 May 14]; 2(1):565-70. Available from: https://www.brazilianjournals.com/index.php/BJHR/article/view/1129

24. Chikhungu LC, Newell ML, Rollins N. Under-five mortality according to maternal survival: a systematic review and meta-analysis. Bull World Health Organ. [Internet]. 2017 [cited 2020 May 19]; 95:281-7. DOI: http://dx.doi.org/10.2471/BLT.15.157149

25. Raine KH, Nath S, Howard LM, Cockshaw W, Boyce P, Sawyer E, Thorpe K. Associations between prenatal maternal mental health indices and mother-infant relationship quality 6 to 18 months' postpartum: A systematic review. Infant Ment Health J. [Internet]. 2019 [cited 2020 May 19]; 41:24-39. DOI: https://doi.org/10.1002/imhj.21825

26. Anjos Am, Gouveia HG. Presence of a companion during the process of labor and childbirth: analysis of practice. Rev enferm UERJ. [Internet]. 2019 [cited 2021 Jun 06]; 27:e38686. DOI: http://dx.doi.org/10.12957/reuerj.2019.38686

27. Andrade LFB, Rodrigues QP, Silva RCV. Good Partices in obstetric care and its interface with humanization of assistance. Rev enferm UERJ. [Internet]. 2017 [cited 2021 Jun 06]; 25:e26442. DOI: http://dx.doi.org/10.12957/reuerj.2017.26442 